\title{
A Scalable GeoWeb Tool for Argumentation Mapping
}

\section{Aaron P. Sani}

Ryerson University

\section{Claus Rinner}

Ryerson University

digital.library.ryerson.ca/object/229

\section{Please Cite:}

Sani, A. P., \& Rinner, C. (2011). A scalable GeoWeb tool for argumentation mapping. GEOMATICA, 65(2), 145-156.

doi:10.5623/cig2011-023

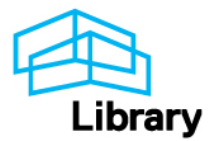




\title{
A Scalable GeoWeb Tool for Argumentation Mapping
}

\author{
Aaron P. Sani, Claus Rinner \\ Department of Geography \\ Ryerson University \\ Email of corresponding author: crinner@ryerson.ca
}

\begin{abstract}
Public participation geographic information systems (PPGIS) support collaborative decisionmaking in the public realm. PPGIS provide advanced communication, deliberation, and conflict resolution mechanisms to engage diverse stakeholder groups. Many of the functional characteristics of Web 2.0 echo basic PPGIS functions, including authoring, linking, and sharing of volunteered geographic information. However, with the increasing popularity of geospatial applications on the Web comes a need to develop concepts for scalable, reliable, and easy-tomaintain tools. In this paper, we propose a cloud computing implementation of a scalable argumentation mapping tool. The tool also illustrates the opportunities of applying a Web 2.0 model to PPGIS. The searching, linking, authoring, tagging, extension, and signalling (SLATES) functions are associated with PPGIS functionality to produce a participatory GeoWeb tool for deliberative democracy.
\end{abstract}

\section{Introduction and Background}

Sieber (2001) characterized Public Participation Geographic Information Systems (PPGIS) as a "normative field of study" that contains "a bottom-up component of public involvement" (p. 2). More broadly, PPGIS support collaborative decision-making processes in the public realm. Using distributed computing technology, PPGIS can facilitate knowledge exchange towards the resolution of ill-defined spatial decision problems (Jankowski and Nyerges, 2001; Balram and Dragicevic, 2006). Ill-defined, or “wicked”, problems are characterized by their inherently ambiguous definitions, objectives, and resolution processes, in addition to the difficulty of determining solutions in anything but qualitative terms (Rittel and Webber, 1973). To facilitate the convergence of opinions towards problem resolution, PPGIS seek to offer various modes of communication to decision-makers in order to develop a common operating picture of the problem domain and its geospatial characteristics. This common operating picture is thought to provide PPGIS users with a joint knowledge base consisting of data relating to the subject matter, which can improve the quality of candidate solutions to the wicked problem. Central to this notion is the engagement of stakeholders, particularly non-GIS savvy laypersons, who participate in online collaboration through highly usable computer interfaces (Jankowski \& Stasik, 1997; Haklay and Tobón, 2003; Sieber 2003).

An argumentation map is a specific example of a PPGIS, one that involves a particularly high level of user contributions through interactions within an online system. The concept of argumentation mapping was proposed by Rinner (2001) to facilitate the geographic referencing of user-generated content (i.e. “arguments”). In the context of Web mapping, the argumentation mapping concept enables linkages between structured discussion and geospatial features, thus 
going beyond annotated maps and map mashups (Rinner et al., 2008; Rinner and Bird, 2009). The major advantages of this approach are twofold. The first advantage is communicative: Stakeholders, whether community-based or from the general public, and independently of their disciplines and roles, can exchange information and ideas with reference to the geographic space under consideration; and they can do this whether geographically dispersed or temporally asynchronized in their ability to communicate. The second advantage is analytical: Decisionmakers and researchers are able to collect and analyze contributions to gain a deeper understanding of how message contents differs spatially as it reflects localized aspects of the decision problem. As such, argumentation mapping provides a conceptual mechanism by which local knowledge and opinions can be incorporated into decision-making.

In their article “Web Mapping 2.0: The Neogeography of the GeoWeb”, Haklay et al. (2008) provide a snapshot of the dynamic development and popularization of geographic information. Recent developments in information technology have enabled a second-generation Web, which can be considered technically as a computing platform and conceptually as a bi-directional communication tool. Web mapping 2.0 and the Geospatial Web, or GeoWeb, allude to the translation of this mainstream IT development to geospatial applications. The bi-directional nature of the GeoWeb allows anyone to contribute "volunteered geographic information" (VGI Goodchild, 2007), in addition to consuming it, and thereby supports the goal of PPGIS to provide access and give voice to people, who were otherwise excluded from many societal decisionmaking processes (Sieber, 2006).

PPGIS projects face a number of interrelated challenges, including issues of data volume, quality, and usefulness; trust between participants; and the duration and depth of user engagement. Sieber (2003) suggests that "scaling up" participation may yield greater engagement. However, the prospect of widespread adoption of PPGIS on the GeoWeb in the context of deliberative democracy raises concerns about the handling of large-scale participation processes (Nyerges, 2005). The quality of user-generated contents is an issue discussed in the literature on VGI. For example, Goodchild (2008) highlights the trade-off between data quality and the economies of scale inherent in low-cost, crowd-sourced data collection. Coleman et al. (2010) discuss engagement from the perspective of contributor motivations in three VGI case studies. Bishr and Mantelas (2008) describe trust as a prerequisite for PPGIS, which in online communities emerges from chains of relations (transitivity), is measured by multiple actors (composability), varies in significance between actors (personalization), and may be asymmetric between any two actors (asymmetry). Elwood (2008) recommends employing user management and auditing to make contributions and user information sufficiently transparent to foster trust within PPGIS.

In this paper, we aim to illustrate the opportunities and ongoing challenges of participatory GeoWeb concepts and tools for engaging people in societal decision-making. We propose a scalable GeoWeb tool for argumentation mapping, which uses a state-of-the-art Web 2.0 deployment approach: cloud computing. In addition, the tool addresses many of the identified PPGIS challenges through Web 2.0 techniques for searching, linking, authoring, tagging, extension and signalling functionality.

In the following Section 2, the technological context of Web 2.0 functionality, implementation, and deployment is discussed. Section 3 proposes a functional framework for participatory GeoWeb tools. Section 4 outlines the cloud computing architecture of Argoomap-GAE and provides a situating scenario used to illustrate the tool's user interface and functionality. The findings of this research are summarized and discussed in the concluding Section 5. 


\section{Technological Context}

The technological context of participatory GeoWeb tools can be differentiated in functional and non-functional characteristics. Therefore, we first describe a detailed model of Web 2.0 functionality, the SLATES model, which will subsequently be associated with participatory GeoWeb functions. Then, we present non-functional aspects of Web 2.0 implementation and deployment, which led to the selection of a cloud computing platform.

\subsection{Web 2.0 Functionality}

The term "Web 2.0" was coined by O’Reilly (2005) to articulate an evolutionary step in the development of the World-Wide-Web. The key vision in Web 2.0 is that the process of Web content creation is opened to the general public. This amounts to 1) enabling public access to existing data through open data layers; 2) providing the public with an unprecedented amount of trust and capability in augmenting data; 3) providing light-weight mechanisms to access and augment the data, and 4) a recognition that the uses of systems developed within this paradigm are "emergent" rather than predetermined. Furthermore, the Web is being transformed into a platform for collaboration and participation, in which 1) users are trusted implicitly as custodians of their data; 2) software is viewed as service components; 3) harnessing the collective intelligence of the masses in the production of new content and/or data enhancement and augmentation is seen as an ideal strategy; and 4) new service creation can be achieved through orchestration of existing services into composite service chains or "mashups" that facilitate data combinations within innovative views.

From a functional perspective, the list of characteristics that constitute a Web 2.0 system proves elusive. There is, however, a related paradigm termed "Enterprise 2.0" that espouses many similar ideals, but in the context of interactions within organizations (McAfee, 2007). McAfee (2007) also propose a model to articulate functionality that is considered essential for Enterprise 2.0. His SLATES model is comprised of requirements for searching, linking, authoring, tagging, extensions, and signalling, which are explained in the following.

Searching refers to the function whereby the contents of an application can be discovered and examined through user-specified search criteria. Searching includes keyword search, full-text search, semantic search, and spatial search, each of which has its own implementation and data requirements. Often, searching is implemented using a keyword search mechanism, in which multiple keywords are associated with user-generated content and can be used to return a reference to the content or resource. Search mechanisms also include the use of statistical indexing of text contents and word-based search that leverages the tagging mechanism described later.

Linking represents to the use of uniform resource identifiers to create associations between content items from disparate systems as a means to promote discovery of related content.

Authoring provides a system with open publishing capabilities whereby users are able to contribute contents to the system. Examples of open content authoring are best appreciated in wikis and blogs, where content develops over time.

Tagging refers to the support of systems for ad-hoc, light-weight, evolving organization. This feature is commonly implemented through user-supplied tag-words ('tags') that are assigned to content items by either the producer of the content item, or another user of the system. By making use of search capabilities of the system, tags can often be used as criteria for the discovery of related content items. As an alternative to explicit searching, graphical components 
known as tag clouds are also used. These components visualize the tags that exist in a content repository, and each tag in the cloud is presented at a size that is in proportion to its prevalence in the system. In this way, popular tags are presented prominently in the tag cloud and the topics available in the repository are communicated.

Extensions refer to the requirement for the system to provide programmatic content access that allows for additional functionality to be created upon existing data. This may include mechanisms that augment core functionality in the system, or mashups that make use of data exposed by the system in addition to data exposed by other systems. By supporting extensions, such systems may facilitate openness and open integration possibilities.

Signaling provides notification mechanisms built on a publish/subscribe paradigm. Examples include Real Simple Syndication (RSS) and GeoRSS services, i.e. RSS that contains a spatial component. The use of these technologies involves the periodic building and publication of an index file that contains links to content. Using tagging functionality, it is possible to subscribe to any subset of the content repository associated with particular keywords. Similarly in the case of GeoRSS services, a user may define a geographic area of interest. If the spatial component of new contents intersects with the spatial extent defined, the user will be notified of the contents' availability.

In addition to the formal SLATES architectural requirements described, there exist other notions tightly associated with Web 2.0. These include social networking and reputation systems, simple but engaging multimedia user interfaces, and support of data mining on social networks and content (Shum, 2008).

\subsection{Web 2.0 Implementation and Deployment}

In order to design a sustainable software system, other aspects beside the high-level usage ought to be taken into account. These aspects are collectively referred to as "non-functional" requirements because they describe constraints and qualities of the system that lie largely outside of the functionality directly sought by end-users. Consideration of the non-functional requirements is essential to providing a successful software implementation (Weigers, 2003). In the context of Web 2.0 implementation, non-functional requirements include (1) system security; (2) performance and scalability; (3) robustness, availability, and fault tolerance; (4) maintainability; and (5) portability, modifiability, and testability.

In the interest of brevity, we will focus on scalability, which was identified as a key concern with participatory GeoWeb applications above. Scalability describes the ability of a system to accommodate increased usage levels through the addition of system resources. Scalability is closely linked to performance, which is typically measured in time units needed to complete a particular functional requirement. For example, a measure of performance could be the number of seconds it takes for a system to output a chart based on thousands of records.

In a typical systems deployment, a software solution is paired with underlying hardware in order to provide acceptable performance for anticipated concurrent usage patterns. If usage patterns change significantly, the hardware capabilities must also improve in order to maintain the performance of the system. If the system is designed with scalability in mind, then the addition of new hardware should result in a corresponding increase in usage capacity.

In a cloud computing environment, 'hardware' is replaced by pools of virtual resources. This allows for faster provisioning of additional resources that may be required to accommodate changing usage patterns. In some cloud computing configurations, additional resource allocation 
is automatic thereby allowing for scalable systems that can provide acceptable performance even during unanticipated usage spikes.

\section{A Functional Framework for Web 2.0-based PPGIS}

The challenges facing widespread online PPGIS adoption that were outlined in the introduction, can be described as follows. Data volumes in a large-scale PPGIS application can create information overload for users, make it difficult to access information of interest, and negatively affect the usefulness and actionability of stakeholder contributions. Large-scale applications may also detrimentally affect the quality of individual content items as well as trust between participants. Independent of scale, the usefulness of a PPGIS can also suffer if its contents is isolated from related information in other existing systems, as well as from fading user interest and ultimately, disengagement, and abandonment.

Table 1 lists these PPGIS challenges and proposes the application of SLATES functionality presented in the technological context to overcome these limitations. Keyword and spatial search functions as well as tagging promote the discoverability of content and thereby encourage use of the system. Linking functions and extensions such as GeoRSS and KML export facilitate integration and depth of information. Authoring mechanisms such as the integration with existing social network accounts promote accountability and trust between users. Finally, signalling functionality provides a way to maintain participant interest and awareness, thereby improving the long-term sustainability of PPGIS applications.

Table 1: PPGIS challenges and Web 2.0 functionality for overcoming them.

\begin{tabular}{|l|l|l|}
\hline PPGIS Challenges & $\begin{array}{l}\text { Web 2.0 Functionality } \\
\text { (SLATES) }\end{array}$ & Benefits for PPGIS \\
\hline Information overload & $\begin{array}{l}\text { Searching: } \\
\text { — Keyword search } \\
\text { - Full-text search } \\
\text { - Spatial search }\end{array}$ & $\begin{array}{l}\text { Promotes discoverability of content; } \\
\text { May encourage use }\end{array}$ \\
\hline $\begin{array}{l}\text { Isolation from other } \\
\text { systems }\end{array}$ & $\begin{array}{l}\text { Linking: } \\
\text { Bi-directional, context- } \\
\text { sensitive linking of } \\
\text { related resources } \\
\text { between systems }\end{array}$ & $\begin{array}{l}\text { Facilitates integration with legacy data; } \\
\text { Promotes depth of information }\end{array}$ \\
\hline $\begin{array}{l}\text { Trust and data } \\
\text { quality; } \\
\text { Disengagement and } \\
\text { abandonment }\end{array}$ & $\begin{array}{c}\text { Authoring: } \\
\text { Integration with existing } \\
\text { social networks }\end{array}$ & $\begin{array}{l}\text { Encourages participation, and openness; } \\
\text { Facilitates learning about contributors as } \\
\text { well as content; } \\
\text { May promote greater sense of } \\
\text { accountability and trust in contributed } \\
\text { content }\end{array}$ \\
\hline
\end{tabular}




\begin{tabular}{|l|l|l|}
\hline Information overload & $\begin{array}{l}\text { Tagging: } \\
\text { Tagging, user-generated } \\
\text { keywords }\end{array}$ & Promotes utility of data and engagement \\
\hline $\begin{array}{l}\text { Isolation from other } \\
\text { Inftems; }\end{array}$ & $\begin{array}{c}\text { Extensions: } \\
\bullet \text { Multiple format exports - } \\
\text { RSS, GeoRSS, KML, } \\
\text { XML }\end{array}$ & $\begin{array}{l}\text { Facilitates integration with external tools } \\
\text { (planning and decision support systems, } \\
\text { mashups); } \\
\text { Provides possibility for summary/digest } \\
\text { data }\end{array}$ \\
\hline $\begin{array}{l}\text { Disengagement and } \\
\text { abandonment }\end{array}$ & $\begin{array}{l}\text { Signalling: } \\
\bullet \text { RSS, GeoRSS }\end{array}$ & $\begin{array}{l}\text { Users stay connected and aware of } \\
\text { engagement of others in their } \\
\text { contributions; } \\
\text { May promote continuous improvement } \\
\text { and interaction }\end{array}$ \\
\hline
\end{tabular}

As a specific type of PPGIS, argumentation mapping tools have been deployed in several case studies designed to examine the utility of the concept in different application domains (Rinner and Bird, 2009; Sidlar and Rinner, 2009; Rinner et al., 2011). The usability of argumentation mapping tools (Sidlar and Rinner, 2007) and their relationship to Web 2.0 concepts (Rinner et al., 2008) was the subject of complementary studies. These experiments have led to successive refinements in the user interface and functionality of the initial "Argumap" prototype (Keßler et al., 2005) and the Google Maps-based “Argoomap” tool (Rinner et al., 2008; Rinner et al., 2011).

With respect to SLATES functionality, all argumentation mapping tools require linking and authoring functions. However, in contrast to the SLATES model, linking has not crossed system boundaries in previous implementations, and authoring has not included modern Web publishing mechanisms such as social networks. The "Argumap” prototype also included limited searching functionality with a full-text search of discussion contributions. Other Web 2.0 functionality from the SLATES model (see Table 1) was not available in argumentation mapping tools prior to the present research.

Other researchers have extended the aforementioned argumentation mapping tools or developed their own. For example, Simao et al. (2009) included Argumap as a component in their wind farm planning tool; Boroushaki and Malczewski (2010) and Meng and Malczewski (2010) extended Argoomap with multi-criteria analysis for local infrastructure planning; and Austerschulte and Keßler (2010) re-implemented Argoomap to include semi-automatic georeferencing of discussion contributions. Through their dependence on the underlying tools, these extensions have the same limitations in implementing SLATES functionality. Furthermore, Zhao and Coleman (2006) and Tang and Coleman (2008) developed and tested a GIS-enabled online discussion forum, "GeoDF”; and Hall and Leahy (2008) and Hall et al. (2010) presented "MapChat" as an asynchronous and synchronous collaboration tool. These PPGIS tools are equally limited in reaping the benefits of SLATES functionality outlined in Table 1. 


\section{Cloud Computing Implementation of an Argumentation Map}

Three cloud computing platforms were evaluated for this project: Amazon EC2, Google App Engine, and Cloud Foundry. We selected Google App Engine because of the initial cost of setup, which is free, the relative simplicity of system deployment, and the integration with Google's social networking facilities. Google App Engine also supports system administration with the monitoring of resource usage performance. This allows the provider to identify costs associated with heavily used system components, and budget accordingly. The following sections describe the architecture of "Argoomap-GAE" and provide examples of its user interface and functionality.

\subsection{Argoomap-GAE Architecture and Use Cases}

The high-level architecture of Argoomap-GAE is shown in Figure 1. The application is deployed within the Google App Engine cloud computing platform and implemented in Java. It is highly modularized within a three-tier architecture including a Web (client) tier, an application service tier, and a data storage tier. Within the Web tier, user interface elements for argumentation mapping are combined with maps retrieved through the Google Maps API. The Web tier also includes components for content export. The application tier contains the components for searching, accessing, and uploading contents to the data repository. It also includes local user management as well as linkages to external user accounts. The only dependency of the application on the Google App Engine is at the level of the persistence store, where the Data Nucleus API is required. To overcome the limitations inherent in using the Data Nucleus API and Big Table to store spatial data, the entire access layer is wrapped as a technology-independent "Spatial Persistence Store", the implementation of which can be modified to accommodate alternate data stores, if the solution was migrated to an alternate cloud platform or a locally administered server. 

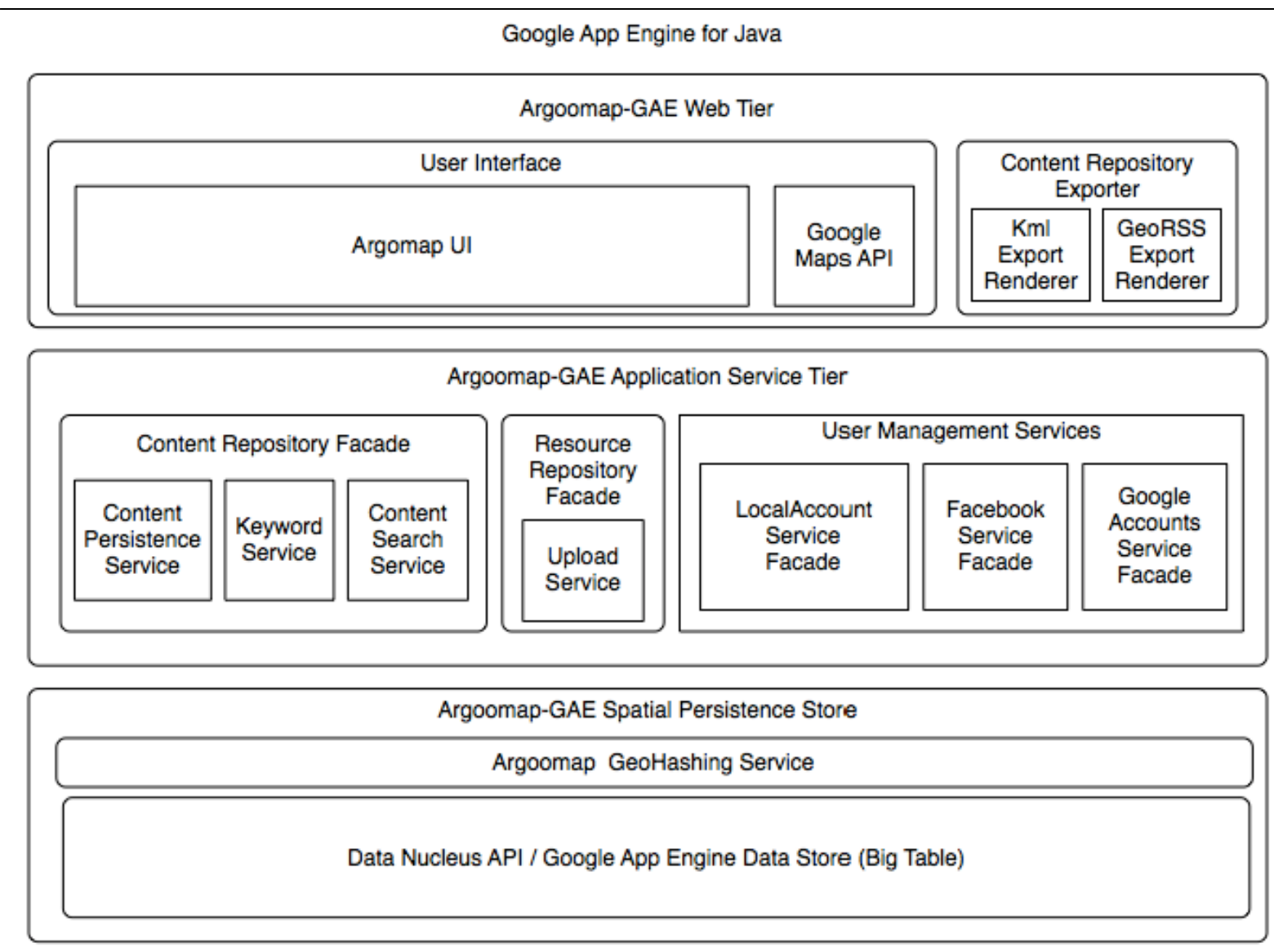

Figure 1: High-level architecture of Argoomap-GAE.

Table 2 lists 18 use cases that describe the intended functionality of Argoomap-GAE, grouped according to the SLATES model. In the following, we describe the correspondence between selected use cases and system architecture components.

Table 2: Argoomap-GAE use cases. Functionality implemented at the time of writing is marked with an asterisk.

\section{Searching}

S1 - find messages related to a spatial extent

*S2 - find messages based on keyword search

*S3 - find messages based on statistical-index text search

\section{Linking}

*L1 - link to external content (video / photo / audio)

L2 - upload binary content (video / photo / audio)

*L3 - post content entered to social networking site Facebook

L4 - send email link of posting to friend

L5 - invite friend to participate 
Authoring (user management)

*A1 - register and login to the application alone

A2 - login using Facebook account

A3 - login using GMail / Google account

\section{Tagging}

T1 - tag content with keyword from "suggested list” of keywords

*T2 - tag content with own keyword

*T3 - explore content via tag-cloud

\section{Extensions}

*E1 - export subset of data as KML for visualization within Google Earth

*E2 - export subset of data as GeoRSS for integration with 3rd party application

\section{Signaling}

SI1 - subscribe to subset of content using keyword criteria and be notified when new content becomes available within the system.

SI2 - subscribe to subset of content using spatial extent and be notified when content becomes available within the system.

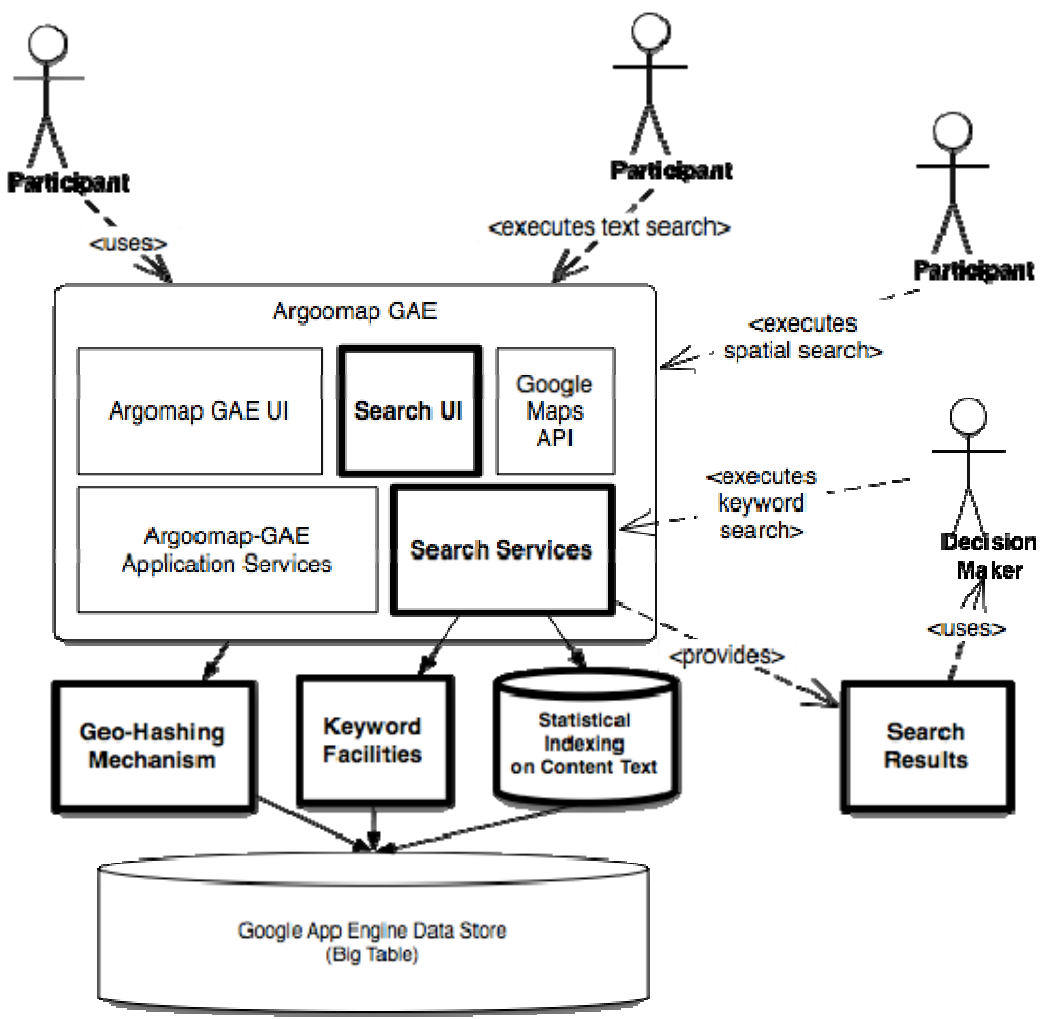

Figure 2: Argoomap-GAE components supporting search functionality

Figure 2 illustrates actors and system components for implementation of the search capabilities. Keyword (text) or spatial search is initiated through a user interface element, which also displays 
the search results. Search is executed through a dedicated service, which provides geo-hashing and keyword facilities and uses automatic statistical indexing of the text of all newly contributed contents. The geo-hashing service is a workaround needed because Google App Engine provides limited support for native spatial data types and queries. Ironically, the technical requirements for statistical indexing were challenging despite the advanced functionality in Google's own search service. Statistical indexing requires the building of a statistical index for all contents in the repository which is updated upon every modification to a content item. Google App Engine does not support such functionality natively and neither do competing platforms. APIs such as Apache Lucene are often employed as an indexing mechanism external to the data store but as its use requires access to a file system, it was not a viable option in Google App Engine. Instead, a derivative of Apache Lucene named Compass API was employed as it provides a workaround to the file system access requirement.

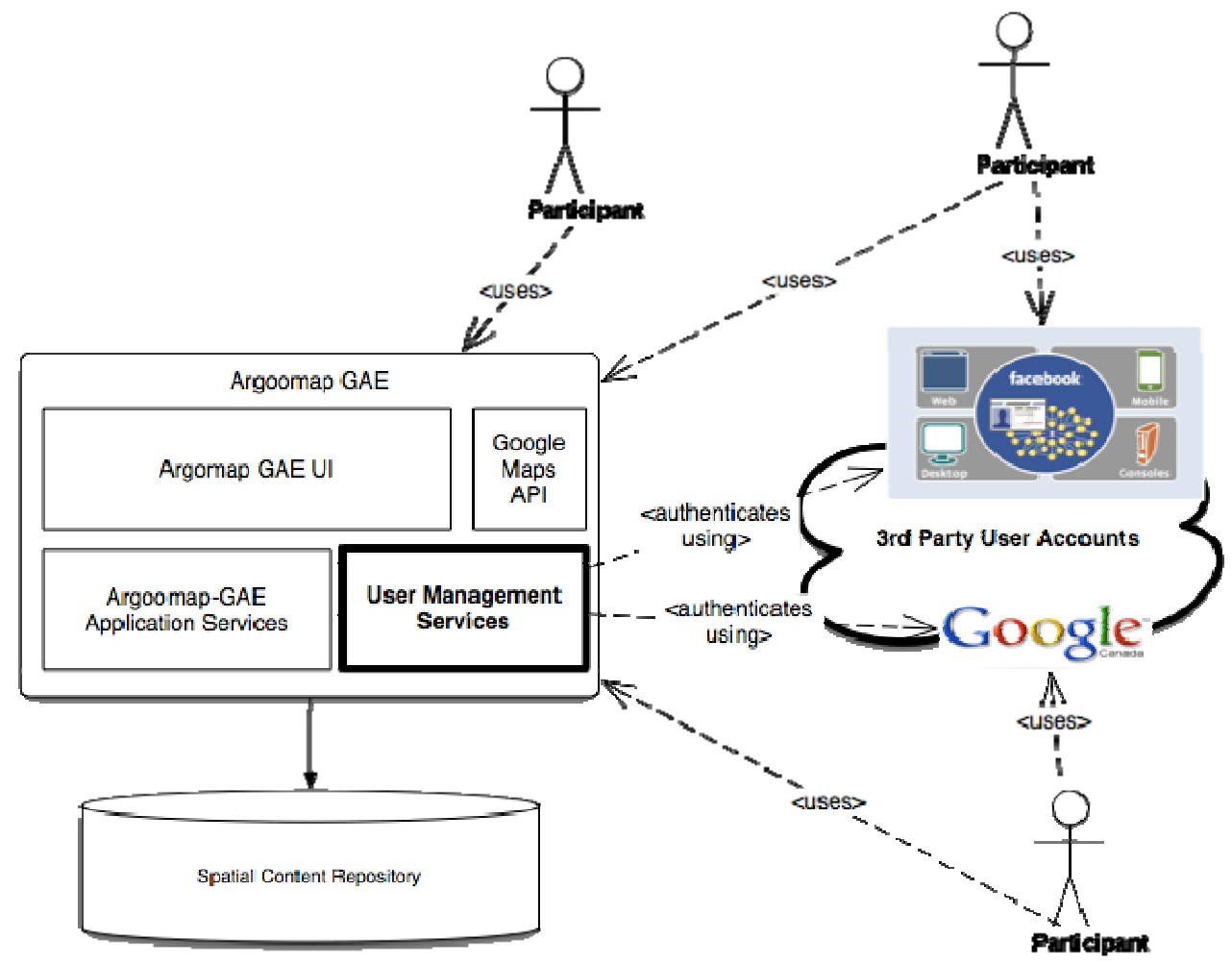

Figure 3: Argoomap-GAE components supporting linking and authoring (user account management)

Components and user interaction for authoring and linking with external social networking software are shown in Figure 3. The user management service allows participants to login through existing accounts using credentials in third-party applications such as Facebook or Google. Once such an authentication has occurred, this user will also be able to link contents from the argumentation map with his or her third-party account. For example, the user may choose to share an Argoomap-GAE contribution with his or her Facebook "friends" by copying it onto his or her "wall”. 


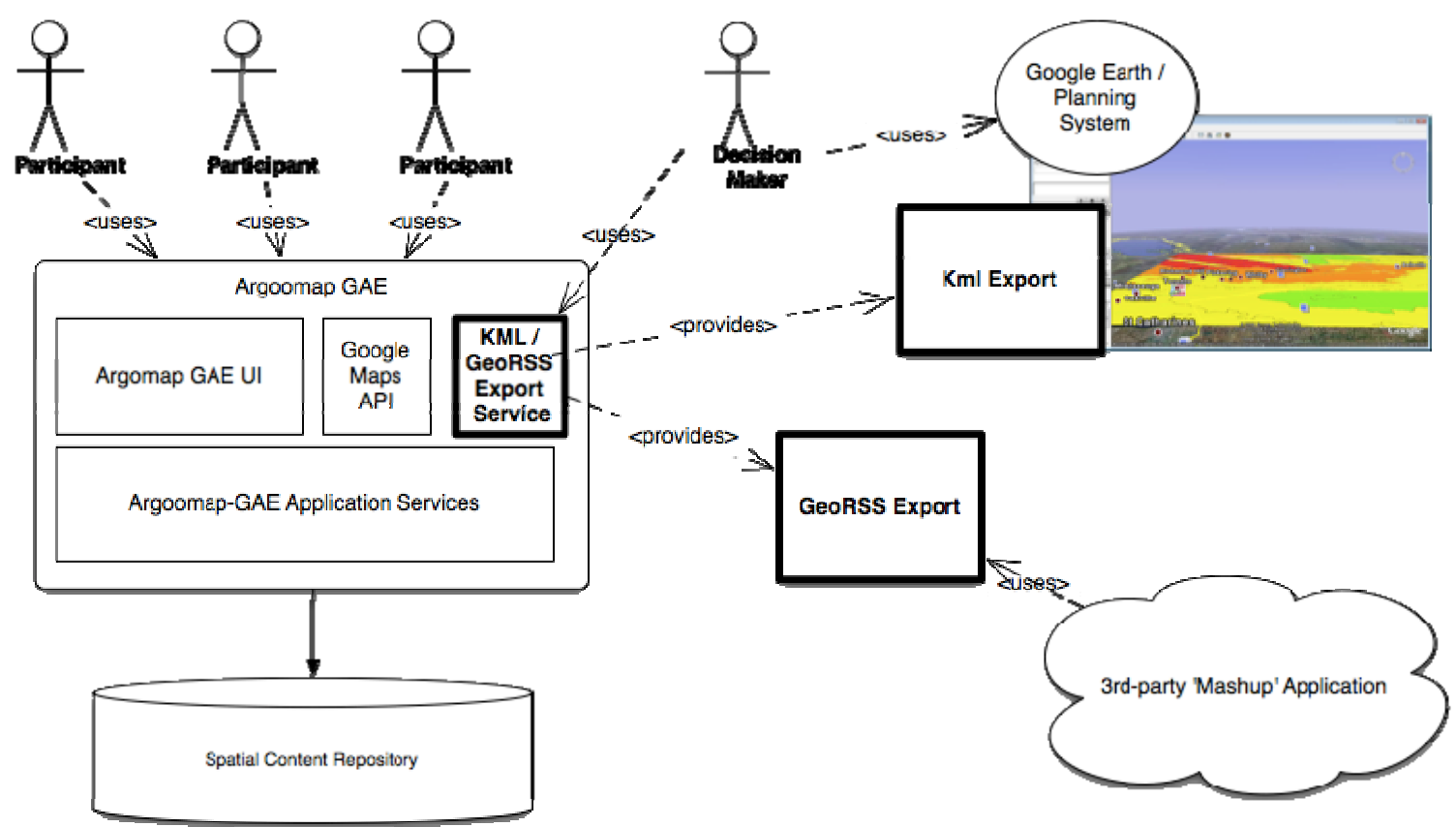

Figure 4: Argoomap-GAE components supporting extensions (export) and signaling functionality

Services related to extension and signaling provide a means to export contents to other applications either once, or continuously. The components highlighted in Figure 4 can generate KML files for transfer to third-party systems such as Google Earth, or create URIs for subscription to an RSS feed of content additions. The technical requirements for the components enabling the extensions and signalling use cases are similar. For performance reasons, rather than providing on-demand access to the entire dataset by an arbitrary number of users, it may be required to limit access to this service to registered users. Furthermore, it may be beneficial to cache the exports as a static files and regenerate the results at a given interval that is configurable and exposed at a known URI.

\subsection{Live Green Toronto situating scenario}

Real-world argumentation mapping case studies conducted by Rinner and Bird (2009), Sidlar and Rinner (2009), and Rinner et al. (2011) involved only small group sizes of 10-20 participants over 1-2 week durations. In contrast, the application envisioned within the Live Green Toronto program may require handling hundreds of almost simultaneous user interactions over an extended period of time. Achieving such high scalability with low technical and budget requirements is the main objective of this research.

Live Green Toronto is an initiative by the City of Toronto to engage residents in greenhouse gas reduction and sustainable life styles. As part of the program, "community animators" were hired to coordinate neighbourhood-level projects. A “sustainability map” is intended to provide mapbased access to neighbourhood activities and a forum for discussion among residents. An argumentation mapping tool is under consideration by the City for the sustainability map. 
Citizen engagement may be fostered by the use of popular and appealing Web 2.0 technology such as Google Maps. The locations of city services, green businesses, non-profit organizations, and neighbourhood initiatives such as community gardens would be used as seed contents in the Live Green Toronto sustainability map. The discussion functionality would support the exchange of new ideas and best-practice approaches between residents, and provide a forum for feedback on local activities.

\subsection{Argoomap-GAE Functionality and User Interface}

The functionality of Argoomap-GAE is illustrated using the Live Green Toronto application scenario. A selection of use cases that were implemented in a preliminary version of the tool are described in the following paragraphs through the corresponding user interface elements.

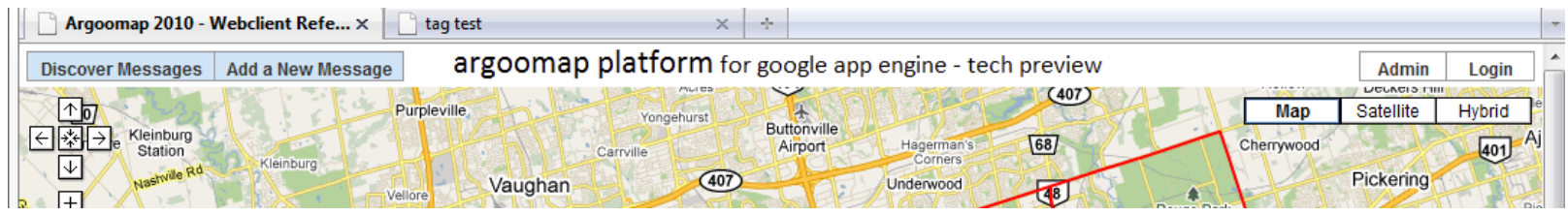

Figure 5: Argoomap-GAE user interface with tabs for message discovery, adding a new message, system administration, and user login.

The Argoomap-GAE user interface consists of a map background that scales to the extent of the browser window and makes use of a panel based layout, in which tab panels are made to appear or disappear by clicking one of four buttons that appear on the top of the user interface depicted in Figure 5.

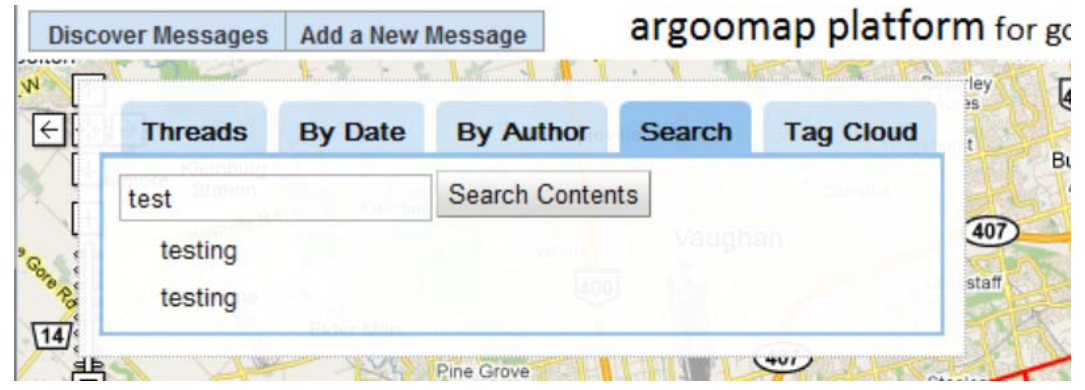

Figure 6: Argoomap-GAE user interface for search functionality.

Users of Argoomap-GAE can access search functionality through the "Search" tab in the "Discover Messages" dialog (Figure 6). By entering search terms and clicking the "Search Contents" button, a user can discover the posted items that contain the submitted keyword(s). Search results are then instantly displayed in the results list below. Users are able to click on an item in the search results and view it in the main window pane. 


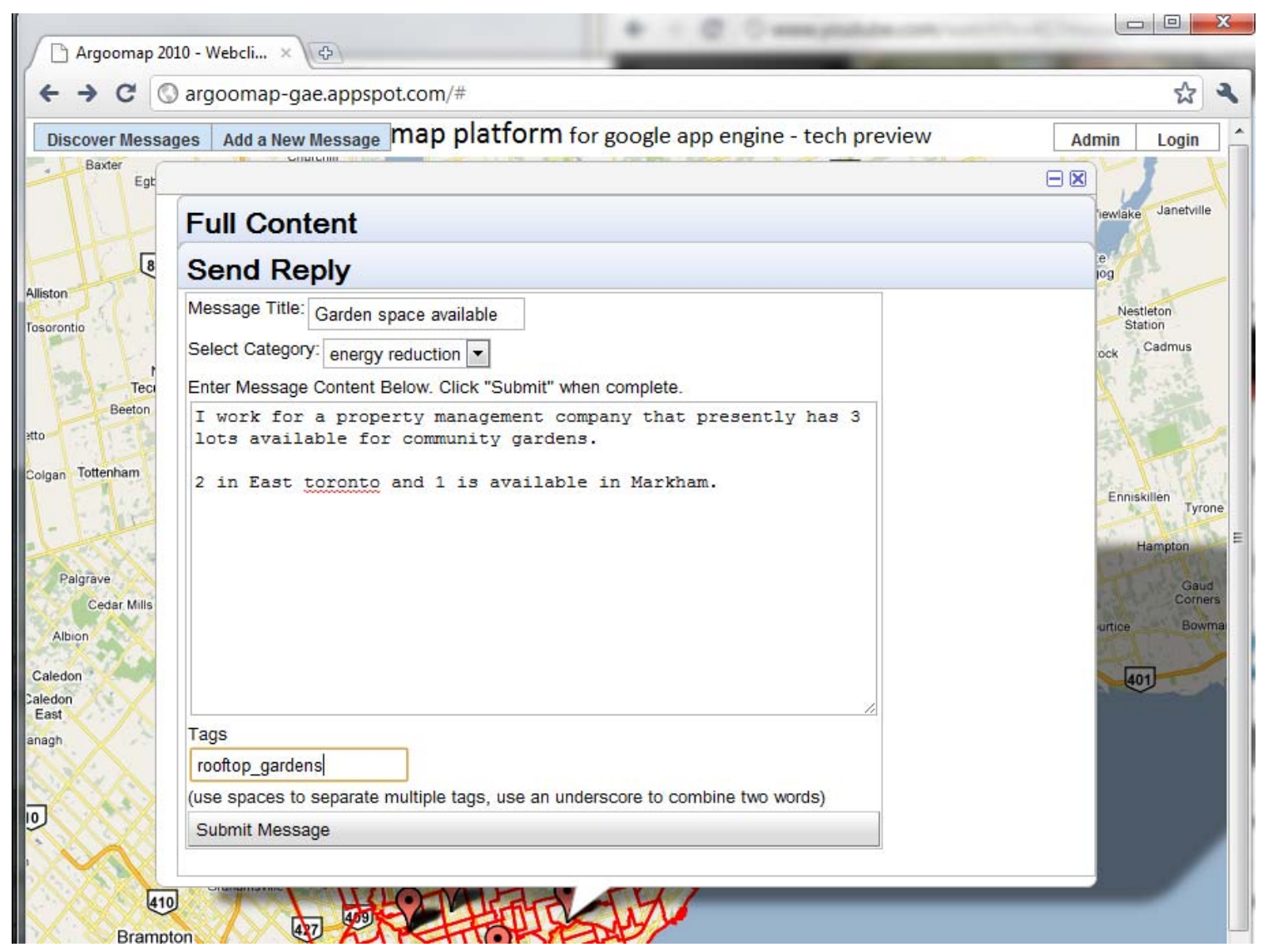

Figure 7: Authoring functionality in Argoomap-GAE

Figure 7 shows the authoring functionality in Argoomap-GAE. Authoring has a three-step workflow associated with it. To identify reference locations, the user clicks a button to enable an edit mode on the map that allows for markers to be added. An additional panel is also opened to allow for a user to exit the edit mode or remove a marker that has been placed incorrectly. Once geographic referencing is completed, a new message window pops up that allows for content entry. When message title, category, content, and tags are populated, the message can be submitted to the system provided that the user has sufficient access rights.

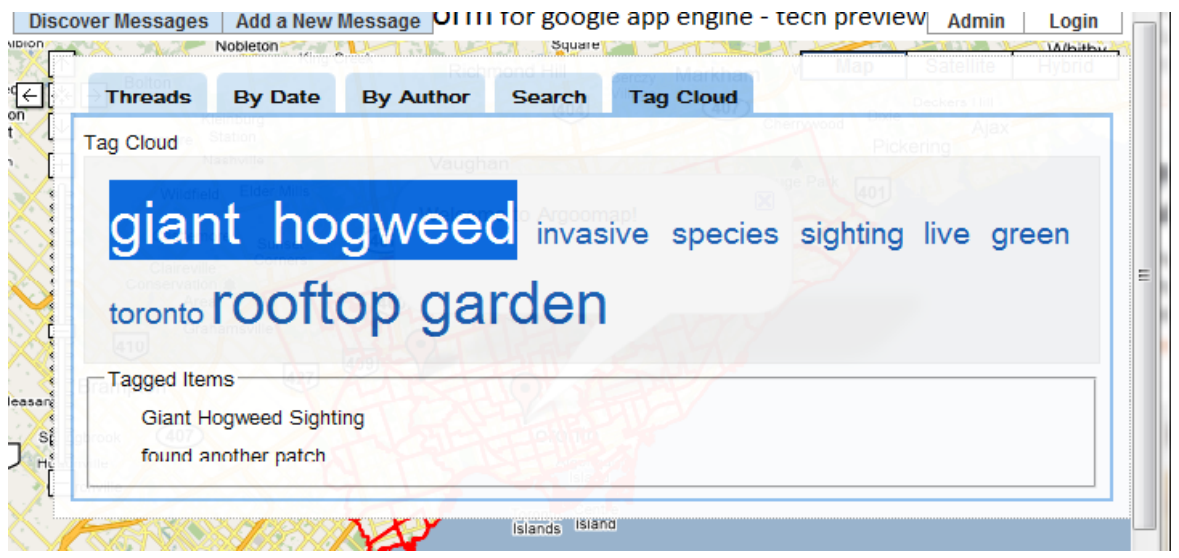

Figure 8: The use of tagging to support content discovery 
The use of tagging for content discovery is illustrated in Figure 8. Multiple tags can be entered as free text in the corresponding text field in the new message dialog (Figure 7). Users are then able to search messages using the tag cloud feature. The tag cloud is rendered in a style similar to the popular Web 2.0 photo sharing application Flickr. When a user moves the mouse over a tag, it is highlighted. If a tag is clicked the user is presented with a clickable list of all contents that has been tagged with the selected term.

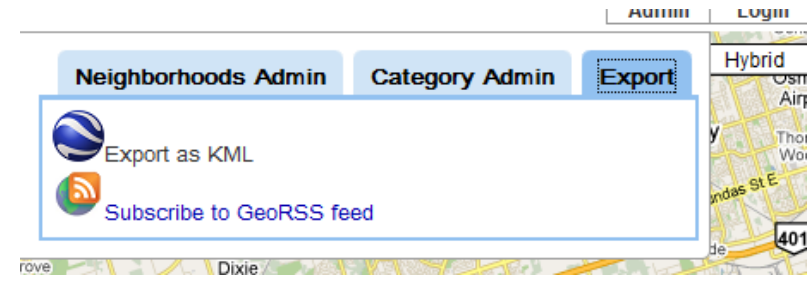

Figure 9: System extension with KML and GeoRSS export functions

Figure 9 illustrates the extension functions of Argoomap-GAE. Messages and their geographic references can be exported as a KML file for use in external applications such as Google Earth. The GeoRSS function provides an RSS export URL, which allows the user to subscribe to the Argoomap-GAE feed using an RSS reader such as Microsoft Outlook. Figure 10 illustrates the automatic signaling by the RSS reader that new content is available on the argumentation map.

\begin{tabular}{|c|c|c|c|c|}
\hline \multicolumn{5}{|c|}{$\notin 83$ GoToMeeting - 29 Meet Now (1) Schedule Meeting = } \\
\hline Mail & $\ll$ & Argoomap GeoRSS & & \multirow{3}{*}{$\begin{array}{l}\text { Giant Hogweed Sighting } \\
\text { Argoomap GeoRSS feed } \\
\text { Click here to view the full article in your default Web browser or to download the article and } \\
\text { any enclosures. }\end{array}$} \\
\hline Favorite Folders & $\hat{\imath}$ & Search Argoomap GeoRSS feed & $\rho \vee \approx$ & \\
\hline Inbox & & Arranged By: Date & Newest on top & \\
\hline Sent Items & & \multicolumn{2}{|l|}{$\boxminus$ Today } & \multirow{3}{*}{$\begin{array}{l}\text { Posted On: Sun 15/05/2011 3:35 PM } \\
\text { Giant Hogweed sighted here: }\end{array}$} \\
\hline $\begin{array}{l}\text { Mail Folders } \\
\text { D] All Mail Items }\end{array}$ & $\begin{array}{l}\hat{\wedge} \\
\vec{r}\end{array}$ & $\begin{array}{l}\text { 며 Argoomap GeoRSS feed } \\
\text { Giant Hogweed Sighting }\end{array}$ & 3:35 PM & \\
\hline 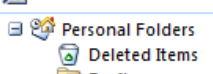 & 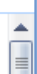 & $\begin{array}{l}\text { Argoomap GeoRSS feed } \\
\text { rooftop garden opportunity }\end{array}$ & 3:23 PM $\square \vee$ & \\
\hline
\end{tabular}

Figure 10: Automatic signalling of Argoomap-GAE contents in RSS reader

\section{Discussion and Conclusion}

The implementation of Argoomap-GAE illustrates the opportunities arising from systematic application of Web 2.0 functionality in the geospatial context. The SLATES functionality represents many of the core functions of PPGIS, such as authoring, linking, and sharing information that are relevant for societal decision-making. The location component in the GeoWeb makes this information more tangible by providing on-the-ground references. Since people tend to be more engaged at the local level than at more abstract levels, GeoWeb tools are drawing considerable interest.

The Live Green Toronto scenario was used to illustrate such a local application. This scenario is characterized by an unknown, but potentially very large number of users with many quasisimultaneous system interactions. While the increased interest in participation on the GeoWeb 
may broaden democratic practices and produce more sustainable solutions in public planning, it also comes with technical challenges. Users of popular tools may experience slow performance and thereby loose interest. Providers of tools in turn will be concerned about dis-engaging users, in particular where participation in societal decision-making is prescribed or desired. We therefore propose a participatory GeoWeb tool for experimentation with cloud computing, a platform that supports scalability by distributing workloads over many computers. The backbone architecture of Argoomap-GAE is conceptually scalable, although a practical test with large user numbers still needs to be conducted.

Additional Web 2.0 features such as social networking were used to facilitate user interaction with the system. For example, an existing Facebook account can be used to sign in to ArgoomapGAE. This feature should make this PPGIS tool more appealing to both, the untrained user who is hesitant to register for any online services and the experienced user who refrains from opening multiple service accounts. The integration with social networking applications also addresses the traditional trust issue in PPGIS by making communication more transparent than a stand-alone tool could do.

We also aimed to provide a freely accessible PPGIS tool that could be easily installed and maintained by community organizations with limited IT support. As an open-source tool, Argoomap-GAE can be set up by anyone and thus introduces a decentralized alternative to corporate social networking sites such as Facebook and Twitter. It could also provide a basis for IT and planning consultancies offering customization and/or installation, operation, and maintenance services. In addition, Google App Engine allows the system administrator to monitor resource use and modify the application accordingly, or pay for use of computing time beyond the allocated free usage.

\section{Acknowledgements}

The authors wish to thank Andrew Millward for his helpful comments on this research. Blake Walker's assistance with finalizing the manuscript is gratefully acknowledged. This research was partially funded by the GEOIDE Network of Centres of Excellence through Project PIV-41 "Participatory Geoweb for Engaging the Public on Global Environmental Change".

\section{References}

Austerschulte, Marius, and Keßler, Carsten (2010) Suggestive Geo-Tagging Assistance for GeoCollaboration Tools. In Painho, Marco, Santos, Maribel Yasmina, and Pundt, Hardy (eds.): Geospatial Thinking. Springer, Berlin/Heidelberg, pp. 143-162

Balram, Shivanand, and Dragicevic, Suzana (2006) Collaborative geographic information systems: Origins, boundaries, and structure. In S. Balram and S. Dragicevic (eds.): Collaborative Geographic Information Systems. Idea Group, Hershey, PA, pp. 1-23

Bishr, Mohamed, and Mantelas, Lefteris (2008) A trust and reputation model for filtering and classifying knowledge about urban growth. GeoJournal 72(3-4): 229-237

Boroushaki, Soheil, and Jacek Malczewski (2010) ParticipatoryGIS: A Web-based Collaborative GIS and Multicriteria Decision Analysis. URISA Journal 22(1): 23-32 
Coleman, David, Sabone, Botshelo, and Nkhwanana, Jairo (2010) Volunteering geographic information to authoritative databases: Linking contributor motivations to program characteristics. Geomatica 64(1): 27-39

Elwood, Sarah (2008) Volunteered geographic information: key questions, concepts and methods to guide emerging research and practice. GeoJournal 72(3-4): 133-135

Goodchild, Michael F. (2008) Commentary: Whither VGI? GeoJournal 72(3-4): 239-244

Goodchild, Michael F. (2007) Citizens as Voluntary Sensors: Spatial Data Infrastructure in the World of Web 2.0. International Journal of Spatial Data Infrastructures Research 2: 24-32

Haklay, Mordechai, and Tobón, Carolina (2003) Usability evaluation and PPGIS: towards a user centred design approach. International Journal of Geographic Information Science 17(6): 577592

Haklay, Mordechai, Singleton, Alex, and Parker, Chris (2008) Web mapping 2.0: the Neogeography of the Geoweb. Geography Compass 2(6): 2011-2039

Hall, G. Brent, and Leahy, Michael G. (2008) Design and implementation of a map-centred synchronous collaboration tool using open source components: the MapChat project. In G. Brent Hall and Michael G. Leahy (eds.) Open Source approaches to spatial data handling. Springer, Berlin, pp. 221-245

Hall, G. Brent, Chipeniuk, Raymond, Feick, Robert D., Leahy, Michael G., and Deparday, Vivien (2010) Community-based production of geographic information using open source software and Web 2.0. International Journal of Geographical Information Science 24(5): 761781

Jankowski, Piotr, and Stasik, Milosz (1997) Spatial Understanding and Decision Support System: A Prototype for Public GIS. Transactions in GIS 2(1): 73-84

Jankowski, Piotr, and Nyerges, Timothy (2001) Geographic Information Systems for Group Decision Making: Toward a Participatory, Geographic Information Science. Taylor \& Francis, London, UK

Keßler, Carsten, Rinner, Claus, and Raubal, Martin (2005) An Argumentation Map Prototype to Support Decision-Making in Spatial Planning. In Fred Toppen and Marco Painho (eds.) Proceedings of AGILE 2005 - 8th Conference on Geographic Information Science, 26-28 May 2005, Estoril, Portugal, pp. 135-142

McAfee, Andrew P. (2006) Enterprise 2.0 - The Dawn of Emergent Collaboration. IEEE Engineering Management Review 34(3): 38

Meng, Yunliang, and Jacek Malczewski (2010) Web-PPGIS Usability and Public Engagement: A Case Study in Canmore, Alberta, Canada. URISA Journal 22(1): 55-64

Nyerges, Timothy (2005) Scaling-Up as a Grand Challenge for Public Participation GIS. Article/comment, Directions Magazine, dated 20 September 2005. Available at http://www.directionsmag.com/articles/scaling-up-as-a-grand-challenge-for-public-participationgis/123341

O’Reilly, Tim (2005) What is Web 2.0? Design Patterns and Business Models for the Next Generation of Software. Available at http://oreilly.com/web2/archive/what-is-web-20.html

Rinner, Claus (2001) Argumentation Maps - GIS-based Discussion Support for Online Planning. Environment and Planning B: Planning and Design 28(6): 847-863 
Rinner, Claus, Keßler, Carsten, and Andrulis, Stephen (2008) The Use of Web 2.0 Concepts to Support Deliberation in Spatial Decision-Making. Computers, Environment and Urban System 32(5): 386-395

Rinner, Claus, and Bird, Michelle (2009) Evaluating Community Engagement through Argumentation Maps - A Public Participation GIS Case Study. Environment and Planning B:

Planning and Design 36(4): 588-601

Rinner, Claus, Kumari, Jyothi, and Mavedati, Sepehr (2011) A Geospatial Web Application to Map Observations and Opinions in Environmental Planning. In S. Li, S. Dragicevic, B. Veenendaal (eds.): Advances in WebGIS, Mapping Services and Applications. CRC Press

Rittel, Horst W.J., and Webber, Melvin M. (1973) Dilemmas in a General Theory of Planning, Policy Sciences 4(2): 155-169

Shum, Simon B. (2008) Cohere: Towards Web 2.0 argumentation. In Proceedings of the 2008 Conference on Computational Models of Argument, pp. 97-108, Amsterdam, The Netherlands. IOS Press

Sidlar, Christopher L., and Rinner, Claus (2007) Analyzing the Usability of an Argumentation Map as a Participatory Spatial Decision Support Tool. URISA Journal 19(1): 47-55

Sidlar, Christopher L., and Rinner, Claus (2009) Utility Assessment of a Map-Based Online GeoCollaboration Tool. Journal of Environmental Management 90(6): 2020-2026

Sieber, Renee (2001) A PPGIScience? cartographica 38(3-4): 1-4

Sieber, Renee (2003) Public Participation Geographic Information Systems across Borders. The Canadian Geographer 47(1): 50-61

Sieber, Renee (2006) Public Participation Geographic Information Systems: A Literature Review and Framework. Annals of the Association of American Geographers 96(3): 491-507

Simao, Ana, Densham, Paul J., and Haklay, Mordechai (2009) Web-based GIS for Collaborative Planning and Public Participation: An Application to the Strategic Planning of Wind Farm Sites. Journal of Environmental Management 90(6): 2027-2040

Tang, Teresa, and Coleman, David J. (2008) GeoDF : Bridging the Communication Gap in Online Participatory Planning. Revue Internationale de Géomatique 18(4): 443-470. Available at http://geo.e-revues.com/article.jsp?articleId=12703

Wiegers, Karl E. (2003) Software requirements. Microsoft Press, Redmond, WA, USA

Zhao, Jianfeng, and Coleman, David J. (2006) GeoDF: Towards a SDI-based PPGIS application for E-Governance. Proceedings of the GSDI 9 Conference, Santiago, Chile, November. Available at http://www.gsdi9.cl/english/papers/TS9.3paper.pdf 